\title{
Technologietransfer kundenorientiert praktiziert
}

\author{
Dipl.-Ing. (FH) Christian Anderson, Dipl.-Ing. (FH) Kay Patzwald
}

Aus dem Bedürfnis heraus, sowohl den Technologietransfer von den Hochschulen in die Unternehmen nicht nur zu begleiten, sondern auch zu praktizieren, als auch praxis- und kundenorientiert zu arbeiten, wurde im Winter des letzten Jahres an der Technischen Fachhochschule Wildau das studentische Projektteam für Technik und Wirtschaft gegruindet, das mit dem Beginn diesen Jahres seine Arbeit aufgenommen hat. Das Team ist dem Technologietransfer- und Weiterbildungszentrum e.V. an der TFH Wildau angeschlossen und kann damit auch in schwierigen Situationen auf kompetenten Ruickhalt zuruickgreifen.

Als Studenten des Aufbaustudiums zum Wirtschaftsingenieur sehen wir unser erstes Betätigungsfeld besonders im Bereich der Ist-Analysen, die sich - angefangen mit der Portfolioanalyse und der Ermittlung von Stärken und Schwächen des Unternehmens - zum einem in der strategischen Unternehmensplanung, und zum anderen im Produktionsbereich wiederfindet in der Schwachstellenanalyse des Produktionsablaufes .

Das zweite Betätigungsfeld ist technischer Natur und befaßt sich neben der Überprïfung von Prozeßautomatisierungsmöglichkeiten besonders mit der Rationalisierung von Prozessen und Abläufen. Da die meisten Aufgaben von einem wirtschaftlich-technischen Dualismus geprägt sind, ergibt sich eine gewollte Kopplung von Technik und Wirtschaft, die wir als besonders herausfordernd empfinden.

Teamarbeit ist fuir uns und in Zusammenarbeit mit dem Kunden ein wichtiges Prinzip. Denn wer komplexe Strukturen und Aufgaben schnell durchdringen will, ist auf sich allein gestellt chancenlos.

Ein weiterer Grundsatz ist es beispielsweise, Aufträge nur zu übernehmen, wenn wir überzeugt sind, daß wir wirklich etwas im Sinne unserer Klienten ausrichten können. Denn wir stellen das Interesse der Klienten über alle anderen Erwägungen.

Zur näheren Konkretisierung unserer Tätigkeitsfelder stellen wir nachfolgend zwei realisierte Praxisfälle vor.

\section{Praxisfall I}

\section{Problem:}

Die monatliche Erstellung der Kennzahlen der einzelnen Betriebsbereiche (elf Stuick) eines großen Unternehmens für Kommunikationstechnik nahm, mit einer Woche, einen erheblichen Zeitanteil der Mitarbeiter in Anspruch. Die Mitarbeiter der Controllingabteilung wurden so mehr durch die Erfassung der Daten belastet als mit der eigentlichen Datenanalyse. Zusätzlich wurde die Erstel- lung durch das tägliche Tagesgeschäft verzögert, von den verständlicherweise auftretenden Fehlern bei der manuellen Übertragung und Eingabe von Daten bzw. dem Kontrollieren der Datenrichtigkeit ganz zu schweigen. Dieser Tatbestand erschwert nicht nur die Arbeit und fordert ein hohes Konzentrationsvermögen vom Ausführenden, sondern läßt die gesamte Abteilung, durch die Aufdeckung von Fehlern, in einem unprofessionellen Licht erscheinen.

Fazit:

Die Erstellung der Kennzahlen benötigte zu viel Zeit und das Vertrauen der Betriebsteilleiter in die Aussagen, Berechnungen und Präsentationen der gesamten Controllingabteilung wurde durch Fehler erschiittert.

\section{Aufgabenstellung:}

Die Erstellung der Kennzahlenblätter sollte rationalisiert und somit mehr Freiraum fuir die Datenanalyse geschaffen werden.

\section{Konzept:}

Quasi auf „Knopfdruck“ werden die entsprechenden Kennzahlen erstellt und per E-Mail an die Leiter der einzelnen Betriebsbereiche versandt. Gleichzeitig wird ein allgemeiner monatsbezogener Datenpool je Betriebsteil angelegt, dessen Datenmaterial auch nachträgliche bereichsübergreifende Vergleiche bzw. neue Kennzahlenauswertungen zuläßt. Aus diesen Datenpoolen ziehen die einzelnen Kennzahlendarstellungen dann die benötigten Daten. Die Kennzahlen enthalten demnach nur auszugsweise die Daten der Datenpoole. Die Kontrolle der Datenrichtigkeit sollte durch ein Abweichungsprotokoll unterstiitzt und effektiv gesteuert werden.
Ablauf:
Die Daten mußten zunächst vom vorhandenen Betriebs- programm (hier SAP) in das Format eines Tabellenkal- kulationsprogramms (Excel) transformiert werden. Um auch im laufenden Jahr nachträglich neue Kennzahlen darstellen zu können, mußte je ein Datenpool je Be- triebsteil erstellt werden. Dieser Datenpool enthält die monatlichen aussagekräftigen Daten eines Betriebs- bereiches sowie wahlweise - wie in diesem Fall - die Daten des letzten Jahres kumuliert, die Plandaten des nächsten und übernächsten. Auf die monatlichen Daten der Datenpoole greifen die meisten Kennzahlenblätter der Betriebsteile zu. Der Datenpool ermöglicht auch noch Monate später die nachträgliche Erstellung neuer Kennzahlen und realisiert damit ein effektives Con- trolling. 


\section{Realisierung:}

1. Zunächst waren die Daten aus SAP-Verdichtungen und BAB-Darstellungen in ein Excel-Format zu transformieren. Das Programm ENTIRE CONNECTION öffnete selbständig Excel, meldete den User in SAP an, rief die gewünschten Kostenverdichtungen auf, uibertrug sie nach Excel und speicherte sie dort in einem „Downloadverzeichnis“. Da beim Öffnen der Verdichtungen die Wahrscheinlichkeit bestand, daß auf bestimmte Bildschirmmeldungen $\mathrm{zu}$ reagieren sei, konnte auf die Anwesenheit von Controllern nicht verzichtet werden. Der sogenannte Download benötigte für die 12 Dateien ca. 5 Minuten.

2. Da jedoch das Makro unbeaufsichtigt laufen sollte, wurde die Abfrage des Abrechnungsmonats, zur Einordnung der Daten, gleich an den Programmanfang gestellt. Für andere Daten, wie zum Beispiel kumulierte Ist-Werte des letzten Jahres, waren in $\operatorname{der} \mathrm{Ab}$ frage vereinbarte Codenummern einzugeben.

3. Von hier an lief das Makro allein. Es öffnete nacheinander die Downloaddateien, formatierte die Daten und kopierte eine Strukturdatei hinein. Diese Strukturdatei enthält für alle Betriebsbereiche die wichtigsten Kostenarten, ein Einpflegen neuer Kostenarten nimmt daher keine nennenswerte Zeit in Anspruch. Anschließend wurden die aktuellen Kostenbeträge in der Downloaddatei als Suchbereich (Datenbank) deklariert. Kostenart um Kostenart durchsuchte das Makro die Downloaddaten. Wurde eine Kosten- oder Leistungsart gefunden, so wurde der Wert übertragen. Wurde eine Kostenart nicht gefunden trug das Programm den Wert „0“ ein. Durch die Strukturdatei wurde gewährleistet, daß, unabhängig vom Vorhandensein der Kostenarten in den Downloaddaten, in den Strukturspalten der Betrag der Kostenart 47110000 immer in der gleichen Zelle steht.

4. Nach dem Einsortieren der Kostenbeträge in die Strukturspalten konnten nun die Beträge aus den Strukturspalten in die Datenpoole uibertragen werden. Dazu wurden nacheinander die Datenpoole der Betriebsbereiche geöffnet und die bestehenden Zellenbezüge aktualisiert. Das Makro trug die in Punkt 2 gemachte Eingabe des Abrechnungsmonats in die entsprechende Spalte ein, in der dann auch die aktuellen Werte eingefügt wurden. Ein Kontrollabgleich wurde vorgenommen. Verglichen wurde die Gesamtsumme aus SAP und die manuell erstellte Gesamtsumme aus den einzelnen Zwischensummen. Diese Kontrolle deckte berechtigte statistische Korrekturen auf, die z. B. bei fehlerhafter Buchung durchgeführt wurden. SAP erfaßt diese Korrektur zwar bei der Zwischensumme, aber nicht bei der Endsumme; daher kann es zu einer Abweichung der Endsumme, gebildet aus den einzelnen Zwischensummen und der Endsumme aus SAP, kommen. Diese Abweichungen - so gering sie auch sein mögen - wurden in ein Abweichungsprotokoll übernommen, das nach dem Durchlauf der Datenpoole automatisch ausgedruckt wird. Der Controller kann sich so effektiv auf die beträchtlichen Abweichungen konzentrieren, ohne sämtliche Dateien öffnen zu müssen.

5. Nach dem Durcharbeiten der Datenpoole folgten die einzelnen Kennzahlenblätter, die jedoch nur ausgewählte Daten aus den Datenpoolen darstellten. Der Ausdruck und/oder Versand der Kennzahlenblätter erfolgte zwar automatisch, jedoch wurden die Blätter vor der Präsentation noch einmal einem fachkundigen Blick unterworfen. Die Möglichkeit, daß Fehler durch das Programm auftreten, sind zwar gering, jedoch ist der menschliche Faktor allein bei der Eingabe von Daten in SAP nicht zu unterschätzen.

\section{Effekt des Programms:}

Die Erstellung der monatlichen Kennzahlen, die durch das Tagesgeschäft bis zu einer Woche in Anspruch nahm, wurde auf $1 \frac{1}{2}$ Stunden reduziert, wobei eine reale Tätigkeits- und Anwesenheitszeit des Controllers von ca. 5 min. benötigt wird. Der so geschaffene Freiraum kann nun effektiv zur Analyse der Kennzahlen in den Betriebsteilen genutzt werden. Reputative Aufgaben sind dem Computer uibertragen worden. Die Einfachheit und Plausibilität des Programms lassen jederzeit Änderungen durch den Controller zu. Durch das Programm konnte die Effektivität der Controllingabteilung wesentlich verbessert werden, da die Zeitersparnis nicht nur über ei-

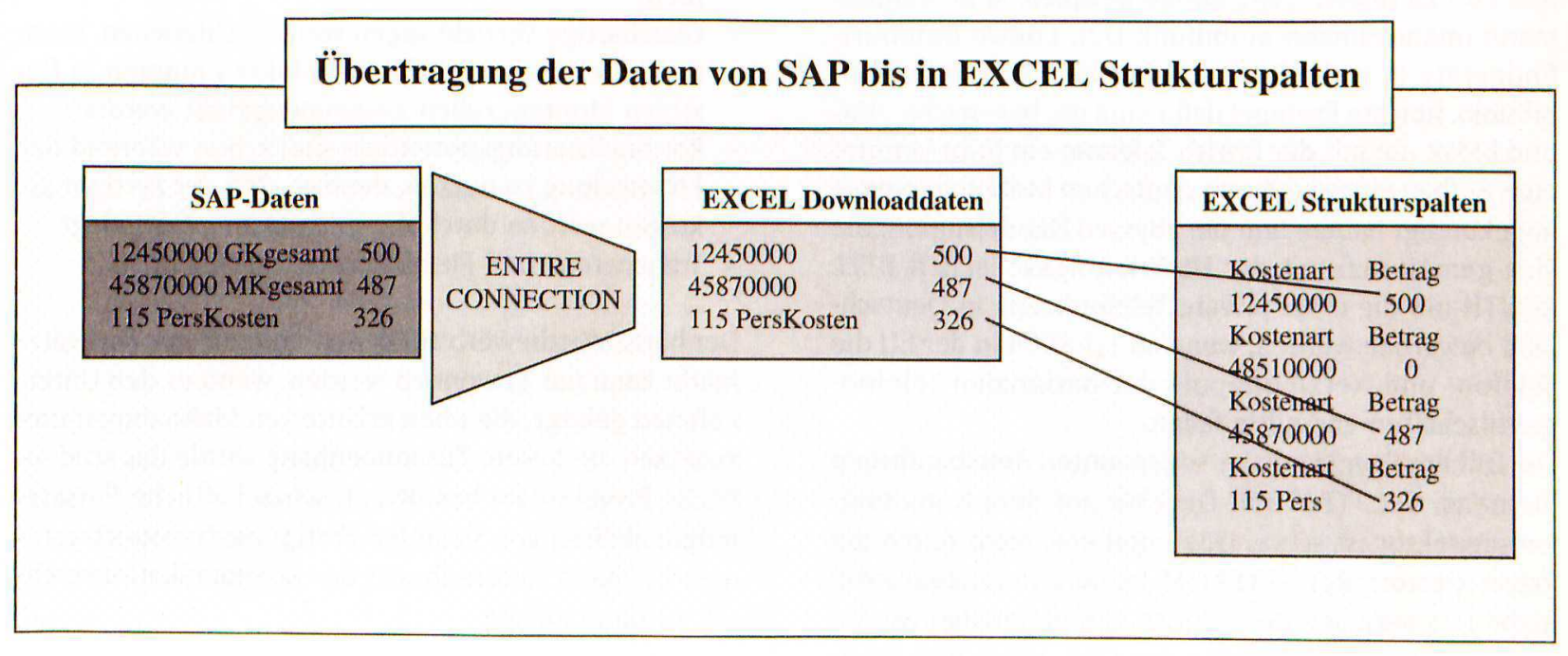


nen Monat, sondern ibber das gesamte Jahr zu sehen ist.

\section{Wirtschaftlicher Nutzen:}

Gemessen an der tariflichen Gehaltsgruppe, die einem Controller gezahlt wird (T 5/6), werden bei vorsichtiger Schätzung im Jahr 13.000 DM zwar nicht direkt eingespart, aber doch zumindest effektiver eingesetzt.

\section{Sicherheit und Kontrolle des Programms:}

In der Testphase wurde das Programm parallel zur manuellen Erstellung auf Fehlerlosigkeit getestet. Dabei traten mehr Fehler bei der manuellen Erstellung als beim Programm auf. Desweiteren konnten die Fehler im Programm (lediglich fehlerhafte Zellenbezüge) schon von den Mitarbeitern der Abteilung selbst behoben werden, was fuir die Simplizität des Programmes spricht.

\section{Ausblick und Kritik:}

Die Schulung der Mitarbeiter im Umgang und Programmieren mit Makros fuihrte schnell zu neuen Einsatzgebieten. Desweiteren konnten wir sicherstellen, daß das Unternehmen durch die Programmeinfuihrung in keine fachliche Abhängigkeit geraten ist, vielmehr konnte den Mitarbeitern ein effektiver und einfacher Weg zur Übertragung reputativer Aufgaben gezeigt werden, den sie mit Freude nutzten. Das Ausführen der Makros schien den Mitarbeitern am meisten gefallen zu haben: zum einen konnten sie den Erfolg ihrer eigenen Arbeit kontrollieren, zum anderen jemandem bei einer sonst langweiligen und wiederholenden Tätigkeit zusehen.

\section{Praxisfall |I}

\section{Einleitung.}

Die achtziger und neunziger Jahre dieses Jahrhunderts sind durch eine zunehmende Liberalisierung der Märkte und eine Verschärfung der Wettbewerbssituation geprägt. Um Kapazitäts- und Strukturkrisen in den eigenen Branchen abzuschwächen und auszuweichen, versuchten viele Firmen durch weitestgehende Diversifikation (Ausweitung des angebotenen Waren- und Produktsortiments) auch in branchenfremde Märkte, wie den Kommunikationsgerätemarkt, einzubrechen und Fuß zu fassen. Langjährige Beispiele sind Mannesmann (mannesmann mobilfunk D2), Loewe (KomfortEndgeräte in vielen Varianten) und VEBA (e-plus mobilfunk). Jüngste Exempel dafür sind die bayerische VIAG und BMW, die mit der British Telekom ein Joint-Venture zum Aufbau eines weiteren deutschen Mobilfunknetzes angekündigt haben, und die Thyssen Handelsunion, die sich gemeinsam mit der US-Telefongesellschaft BELL SOUTH um die erste private Telefonlizenz in Deutschland bewerben werden, wenn ab 1.1.1998 in der EU die Telefon- und Netzmonopole der nationalen Telefongesellschaften endguiltig fallen.

Die fruihere Dominanz der sogenannten Amtsbaufirmen Siemens, SEL, T\&N und DeTeWe auf dem Kommunikationssektor ist schon lange und erst recht durch die Privatisierung der TELEKOM keine Existenzgarantie mehr. Das permanente Drängen von nationalen wie internationalen Unternehmen aus USA, Japan und Asien auf den deutschen Kommunikationsgerätemarkt verschärft zunehmend den Wettbewerb in Deutschland. Hohe Löhne aber vor allem die tariflichen Lohnfolgekosten von $80 \%$ und mehr, bilden im Hochlohnland Deutschland eine schwache Ausgangsposition für einen umkämpften Markt. Will man den Markt nicht den uibrigen Mitbewerbern uiberlassen, muissen andere Maßnahmen die Wettbewerbsfähigkeit wieder herstellen, wie zum Beispiel

- High-Tech-Produkte,

- hohe Qualität der Produkte,

- Verringerung der Fertigungstiefe,

- eine wirtschaftliche und flexible Automatisierung,

- geringe Lagerbestände bei hoher Verfuigbarkeit und

- geringe Durchlaufbestände.

Um Deutschland als Fertigungsstandort zu erhalten, muß es den deutschen Unternehmen gelingen, ihre Produkte und ihre Fertigung international konkurrenzfähig zu gestalten, dazu gehört auch die Übernahme von einem in Japan entwickelten Fertigungssteuerungsprinzip KANBAN bzw. JUST-IN-TIME.

Die Notwendigkeit, dem Markt heute komplette Produktsysteme zur Verfügung stellen zu müssen, die ihrerseits einem ständigen technologischen Wandel unterworfen sind, fuihrt zu einem Produktspektrum, das hohe Anforderungen an die Flexibilität der Produktion von morgen stellt. In diesem Sinne gilt es, Produkt und Produktionsbedingungen anzupassen, denn eine Montage - wie fruiher - nur für ein eng definiertes Produktspektrum zu planen, ist wegen der immer kürzer werdenden Innovationszyklen und der Technologiesprünge nicht mehr geeignet.

Da Markt-, Produkt-, und Technologieentwicklungen nur mit zunehmender Unsicherheit bei der Gestaltung von Montagesystemen miteinbezogen werden können, kann keine direkte Gestaltungsrichtung angegeben werden. Dennoch können einige wichtige Thesen formuliert werden:

- Die Montage von einfachen Standardanlagen ist im Hochlohnland Deutschland wirtschaftlich kaum mehr möglich.

- Das verbleibende technologisch hochentwickelte Produktspektrum hat ein wesentlich kleineres Volumen.

- Gleichartige Verrichtungen von verschiedenen, montagegerecht entwickelten Produkten müssen in flexiblen Montagezellen zusammengefaßt werden.

- Rationalisierungspotentiale sind schon während der Entwicklung zu nutzen, denn ca. 70\% der Fertigungskosten werden durch die Entwicklung festgelegt.

- Transparenz und Flexibilität der Fertigung.

Der harte Wettbewerb auf dem Kommunikationsgerätemarkt kann nur gewonnen werden, wenn es den Unternehmen gelingt, die oben erläuterten Maßnahmen umzusetzen. In diesem Zusammenhang wurde das studentische Projektteam beauftragt, wirtschaftliche Einsatzmöglichkeiten von flexiblen Fertigungstransportsystemen in einem Unternehmen der Kommunikationstechnik zu untersuchen. 


\section{Aufgabenstellung:}

Zu untersuchen war die Möglichkeit der Zusammenlegung von mehreren Montagelinien (drei Linien) und der Rationalisierung bzw. Automatisierung der einzelnen Prozesse durch den Einsatz flexibler Fertigungstransportsysteme.

Was sind flexible Fertigungstransportsysteme (FTS)? FTS gewährleisten durch die Verbindung der verschiedenen aufeinanderfolgenden Arbeitsplätze die Fließfertigung und damit minimale Aufenthaltszeiten bzw. Lagerzeiten der Produkte innerhalb der Montagelinie. Ein so gestalteter Prozeß läßt sich wie folgt darstellen: Am Montageplatz werden Teile zu einer Baugruppe montiert, dabei dient bereits der Werkstuickträger (WT) als Montagevorrichtung, dieser WT kann sich entweder bereits auf dem Transportband befinden oder aber der Mitarbeiter legt die Baugruppe in den WT ein, der sich in einer Ausschleusung befindet. In jedem Fall erfolgt auf Knopfdruck nun die Freigabe des WT's, und nach einem im Hauptrechner hinterlegten Fertigungsablauf wird der WT direkt zum nächsten Arbeitsplatz, bei mehreren verfügbaren zum nächsten freien Arbeitsplatz, gesteuert. Ausschleusungen aus dem Hauptkreislauf dienen dazu, den schnellen Transport der WT's zu garantieren, desweiteren erfüllen sie bei großen Fertigungslinien die Funktion eines Puffers. Dem Fertigungsablauf folgend, wird das Produkt dann zum Prïfen gesteuert.

Bei einem negativen Test erfolgt der Transport zur Reparaturstation, ansonsten wird der WT zum Verpakken gesteuert. Jeder WT trägt eine Transpondernummer, mit der im Hauptrechner, in Abhängigkeit vom Arbeitsplatz, von wo aus der WT abgeschickt wurde, ein bestimmtes Produkt verknüpft wird. Für jedes Produkt ist ein definierter Fertigungsablauf hinterlegt, nach dem die WT's gesteuert werden. Leere WT's können, wenn es die Produktformen erlauben, universell eingesetzt werden, ansonsten ist es auch möglich, die Werkstïckträger nur einem einzigen Produkt zuzuordnen.

In jedem Fall kann durch ein Fertigungstransportsystem die Chaosfertigung realisiert werden, d. h. mehrere verschieden Produkte (z. B. Telefon, Fön und Computermonitore) können ohne Probleme zum Teil die gleichen Fertigungswege benutzen, was den Platzbedarf enorm reduziert. Zusätzlich können zur besseren Auslastung bestimmter Tätigkeiten, Arbeitsplätze zusammengefaßt werden, d. h. zum Beispiel, daß der Verpackungsvorgang von zwei verschiedenen Produkten an dem selben Platz vollzogen werden kann.

Ein weiterer Vorteil liegt in der Automatisierung von einfachen Vorgängen wie in diesem Fall des Prïfens. Die Werkstuickträger bieten hervorragende Möglichkeiten für Aufnahmen von Prüfanschliissen, Kontaktierungen, definierte Lagen und ähnliches. Koppelt man dann noch die Prüfeinheiten mit einem Tintenstrahldrucker, werden alle gut-geprüften mit aufeinanderfolgenden Seriennummern versehen. Obwohl das folgende mehr die Prïftechnik betrifft, soll es hier trotzdem Erwähnung finden.

Zu jedem schlecht-gepriften Produkt wird der Werkstückträgerkennung ein Prïfprotokoll zugeordnet. Dieses Protokoll wird mit der Werstiickträgernummer ver- kniipft und im Netz hinterlegt. Am Reparaturplatz kann dieses Protokoll zum jeweiligen Produkt am Bildschirm abgerufen und der Fehler behoben werden, effektiv und vollkommen papierfrei. Desweiteren können die Prüfprotokolle nun weitaus differenzierter sein, da sich der Umfang der Darstellung an einem Bildschirm wesentlich vergrößert hat.

Zusammenfassend lassen sich folgende Vorteile von FTS-Linien nennen:

- geringer Platzbedarf durch den kombinierten Gebrauch der Fertigungswege,

- sofortiger Transport von bearbeiteten Baugruppen zum nächsten Arbeitsplatz (geringe Durchlaufzeiten minimale Zwischenlagerbildung),

- Automatisierungsmöglichkeit von Fertigungsabschnitten,

- die Mitarbeiter sind nicht mehr fuir den Transport der Baugruppen zum nächsten Arbeitsplatz verantwortlich,

- Realisierung der Fließfertigung,

- Fertigung nach dem Bringe-Prinzip.

Dem Leser mögen diese Vorteile als geringwertig erscheinen, doch machen sie, wie in diesem Fall nachhaltig dargestellt wird, den Hauptteil der Einsparungen aus.

\section{Konzept:}

\section{Aufinahme des Ist-Zustandes}

Eine Kapazitätsüberprüfung ergab, daß für die Stückzahlen im laufenden Jahr von den 12 beschäftigten Mitarbeitern lediglich 6 effektiv unter Beruicksichtigung von Planungsfaktor, Zeitgrad und Krankenstand benötigt wurden.

(Anmerkung des Projektteams: Da die Gründe für das untertriebene Kapazitätsangebot angesichts der herrschenden Wirtschaftslage zwar moralisch, aber nicht betriebswirtschaftlich nachvollziehbar waren, wurde dem Kostenstellenleiter empfohlen, von sich aus die übrigen Mitarbeiter den anderen Abteilungen zur Verfuigung zu stellen.)

Auch bei der Analyse der Arbeitspläne und deren Umsetzung konnten Mängel festgestellt werden, gleiche Verrichtungen wurden bei den Produkten mit unterschiedlichen Zeiten und Lohngruppen bewertet. Ebenso traten Mängel bei der montagegerechten Gestaltung der einzelnen Produkte und der Einrichtung der Arbeitsplätze auf.

\section{Soll-Zustand}

Die Priufung der Produkte kann durch ein flexibles Montagesystem automatisiert werden. Das System übernimmt auch sämtliche Transporte der Produkte von und zu den einzelnen Arbeitsplätzen der Fertigungslinie. Desweiteren konnte der Verpackungsvorgang in die Linie integriert werden, was das gesammelte Transportieren der Produkte zum Verpackplatz erübrigte. Der nur schwach ausgelastete Reparaturplatz wurde zwischen der neuen und einer weiteren Fertigungslinie plaziert, um seine effektive Auslastung zu sichern. Es konnte ein Aufgabenwechsel zwischen der Montage und dem Verpacken eingefuihrt werden. Der Raumbedarf konnte dra- 


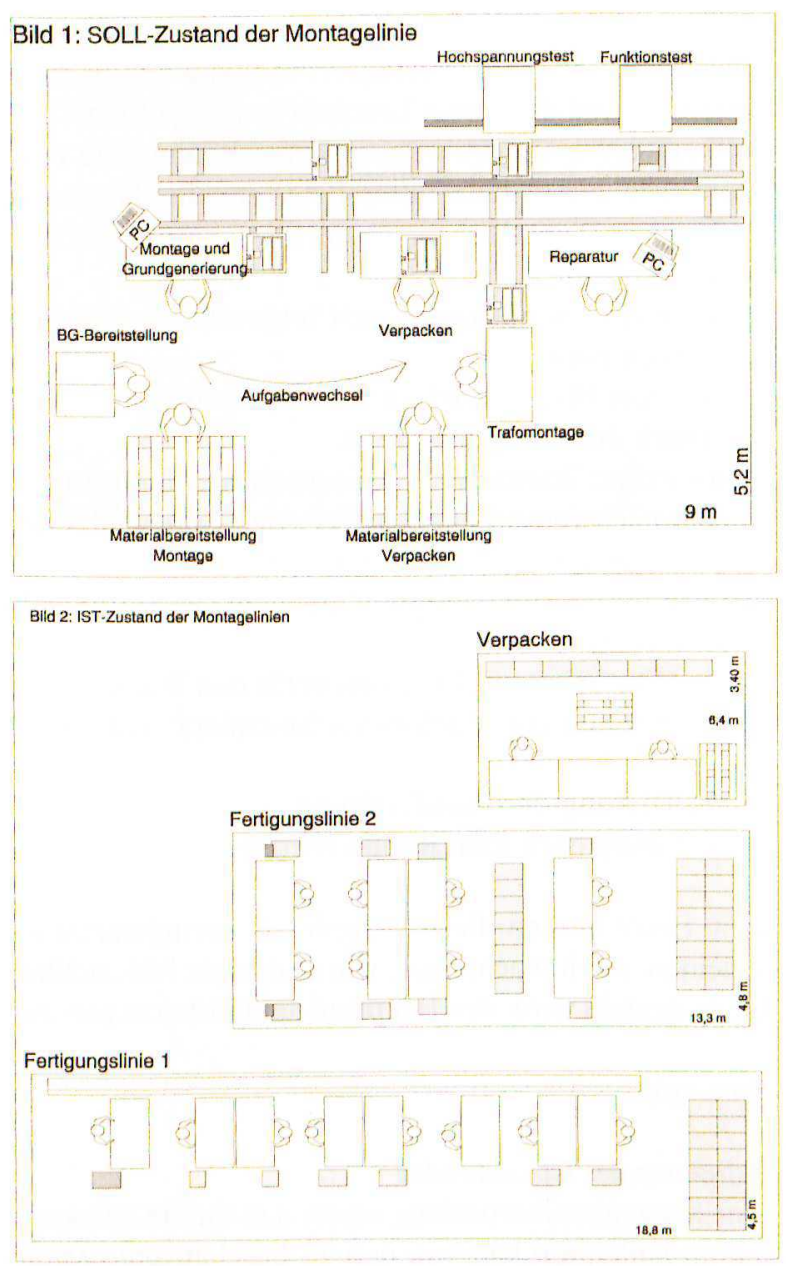

stisch gesenkt werden (vergleiche Bild 1 „Soll-Zustand“ mit Bild 2 „Ist-Zustand“).

Ergebnisse:

Insgesamt wurden und können durch die Investition in ein neues Transportsystem folgende über fünf Jahre abgezinste Einsparungen gemacht werden:

Einsparung durch die Überarbeitung der Arbeitspläne (wurde durch die Analyse aktiviert) Einsparung von Fertigungslohn und Lohnfolgekosten durch Rationalisierung und Automatisierung

Einsparung von Gemeinkosten

\section{Summe}

794.145 DM

Summe (abziiglich der Investsumme von $120.000 \mathrm{DM})$ einzelnen Abteilungsmitglieder Logistik und Produktionsunterstiitzung angetan.

Dennoch hätten wir von der Geschäftsleitung mehr Offenheit in Bezug auf die jährlichen Stuickzahlen erwartet. Uns ist zwar klar, daß dies meistens einen sensiblen Bereich der Fertigung darstellt, doch kann eine Analyse nur so gut wie das Ausgangsmaterial sein, möglicherweise geschätzte Stückzahlen und Hinweise wie „Nehmen sie doch einfach 'ne Zahl an" helfen hier nicht weiter.

\section{Verfasser}

Dipl. Ing. (FH) Christian Anderson

Dipl. Ing. (FH) Kay Patzwald

Technische Fachhochschule Wildau

Studentische Unternehmensberatung NOVACON Tel. (0 33 75) 508-912

Die Investitionssumme beträgt für das Transportsystem 120.000 DM.

Die dynamische Amortisationsdauer beträgt rund 1,5 Jahre.

Der interne Zinsfuß der Investition liegt bei $44,25 \%$.

Desweiteren könnte durch die Investition $72 \%$ des bisherigen Platzbedarfes eingespart werden. Durch die Rationalisierung werden $22 \%$ der Kapazität pro Jahr eingespart bzw. wurde eine Produktivitätssteigerung von $28 \%$ erzielt.

\section{Kritik und Ausblick:}

Trotzdem eine Analyse meistens nur mögliche Einsparungen in Aussicht stellt, waren wir doch erleichtert durch die Analyse der Arbeitspläne und Prozesse eine Einsparungssumme von ca. 250.0000 DM aktivieren zu können. Desweiteren waren wir von der Mitarbeit der 